INRA Prod. Anim., 2007, 20 (3), 199-206

\title{
Emergence des maladies infectieuses animales et humaines
}

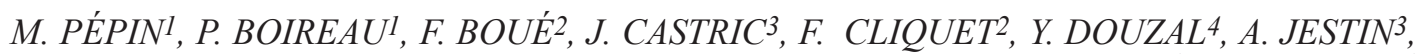 \\ F. MOUTOU ${ }^{1}$, S. ZIENTARA ${ }^{1}$ \\ 1 AFSSA, LERPAZ, 23 avenue du Général de Gaulle, F-94701 Maisons-Alfort, France \\ 2 AFSSA, LERRPAZ, Domaine de Pixérécourt, BP 9, F-54220 Malzéville, France \\ ${ }^{3}$ AFSSA, LERAPP, BP 53, F-22440 Ploufragan, France \\ ${ }^{4}$ AFSSA, Direction scientifique, 21-27 avenue du Général Leclerc, F-94706 Maisons-Alfort, France \\ 1, 2, 3, 4 Groupe «Maladies Infectieuses Emergentes» de l'AFSSA \\ Courriel:m.pepin@afssa.fr
}

\section{1 / Définition d'une maladie émergente}

Il n'existe pas de réel consensus autour d'une définition universelle du concept de maladie émergente, à tel point que divers organismes internationaux ont adopté une définition différente. Pour cette revue, nous avons retenu la définition adoptée par la Cellule Permanente des Maladies Infectieuses Emergentes (CP-MIE) mise en place sous l'égide du Ministère de la Recherche en 2006 : «Une maladie infectieuse émergente est un phénomène infectieux (ou présumé comme tel) inattendu - en référence à ses propriétés intrinsèques ou les connaissances de sa biologie - touchant l'homme, l'animal ou les deux. Il peut s'agir d'une entité clinique d'origine infectieuse nouvellement apparue ou identifiée, d'une entité pathologique infectieuse connue dont l'incidence augmente dans un espace ou dans un groupe de population donné ou d'une modification qualitative et/ou quantitative des caractéristiques de l'agent, de la maladie ou de la population touchée et de son environnement. Dans une optique d'anticipation, il peut s'agir d'une maladie identifiée dont les conditions d'expression deviennent favorables. Habituellement, une incertitude réelle ou perçue quant au potentiel évolutif, quant à la maîtrise du phénomène et de son impact en santé publique humaine et/ou animale, est présente».

Cette définition permet de faire ressortir quelques constantes dans les définitions des maladies émergentes : augmentation assez brutale de l'incidence dans le temps et/ou dans l'espace, conditions d'expansion modifiées et incertitude quant à l'ampleur du phénomène nouveau.

Ce concept de maladie émergente est ancien puisque Charles Nicolle avait déjà cité en 1933 cette notion dans son ouvrage intitulé «Naissance, vie et mort des maladies infectieuses» (Toma et Thiry 2003).

Parmi les nombreux exemples de maladies émergentes récentes, il est possible de faire la distinction entre :

- les maladies dont la cause est effectivement nouvelle : c'est le cas de la maladie de lyme due à Borrelia burgdoferi, le SRAS ou Syndrome Respiratoire Aigu Sévère apparu en Chine en novembre 2002, l'infection par le virus de l'immunodéficience humaine apparue au début des années 80 ou encore l'ESB et la MCJnv chez les bovins et l'Homme respectivement ;

- les maladies dont la cause était connue jusqu'alors et dont l'incidence augmente considérablement (parce que souvent apparaissant dans un endroit, une région, un pays où l'agent pathogène était inconnu jusqu'à l'émergence) : les viroses dues au virus West Nile en Amérique du Nord ou plus récemment le virus du Chikungunya (Josseran et al 2006) ou le virus de la fièvre catarrhale ovine (bluetongue) dans le Nord de l'Europe (Toussaint et al 2006), ou des parasitoses comme la trichinellose en Roumanie après le changement politique des années 90 (Blaga et al 2007), pour ne citer que des exemples récents et médiatisés.

Il est important de mentionner la notion d'émergence médiatique qui amplifie le phénomène auprès du public et auprès des décideurs (phénomène que nous pourrions qualifier de «caisse de résonance») et contribue ainsi à faire «émerger» un phénomène déjà bien identifié auparavant, avec l'exemple très instructif de la listériose puisque c'est bien l'écho médiatique qui a fait passer la listériose du statut de maladie importante certes, mais plutôt confidentielle, à un statut de maladie majeure alors que l'incidence a peu varié au cours du temps (Toma et Thiry 2003).

\section{2 / Caractéristiques des agents pathogènes émer- gents}

En focalisant leur analyse sur les agents pathogènes humains, Woolhouse et Gowtage-Sequeria (2005) ont récemment recensé 1407 agents infectieux (liste disponible sur le site suivant : www.cdc.gov/ncidod/eid/ vol11no12/05-0997 app.htm) dont 177 peuvent être considérés comme émergents et réémergents (soit 13\%). Le tableau 1 donne les répartitions de ces 1407 agents pathogènes parmi les 5 grandes familles d'agents pathogènes : les virus regroupent ainsi de façon significative plus d'agents émergents ou réémergents en comparaison avec les autres groupes : bactéries, champignons, parasites, etc. Parmi les virus, les familles les plus représentées sont les Bunyaviridae, les Flaviviridae, les Togaviridae et les Reoviridae (Heeney 2006).

Il faut noter que parmi les 177 agents infectieux émergents, 130 soit $73 \%$ sont des agents zoonotiques, c'est-àdire transmissibles de l'animal à l'Homme ; une fois transmis à l'Homme, ces agents zoonotiques sont 
Tableau 1. Nombre d'agents pathogènes connus et émergents pour l'Homme (Woolhouse et Gowtage-Sequeria 2005).

\begin{tabular}{|c|c|c|c|}
\hline Groupe ou entité & $\begin{array}{c}\text { Nombre d'agents } \\
\text { pathogènes } \\
\text { connus à ce jour }\end{array}$ & $\begin{array}{c}\text { Nombre d'agents } \\
\text { émergents ou } \\
\text { réémergents }\end{array}$ & $\begin{array}{c}\text { Pourcentage } \\
\text { (\%) }\end{array}$ \\
\hline Virus et prions & 208 & 77 & 37 \\
Bactéries & 538 & 54 & 10 \\
Champignons & 317 & 22 & 7 \\
Protozoaires & 57 & 14 & 25 \\
Helminthes & 287 & 10 & 3 \\
Total & 1407 & 177 & 13 \\
Agents zoonotiques & 816 & 130 & 16 \\
\hline
\end{tabular}

pour la plupart d'entre eux non transmissibles d'homme à homme, l'homme jouant le rôle dans ce cas de «culde-sac» épidémiologique (à l'exemple de la rage, du virus de la Rift Valley Fever...). Seule une minorité est fortement transmissible $(10 \%)$ ou faiblement transmissible ( $25 \%$ environ) de personne à personne. Si les Ongulés représentent la principale source d'agents pathogènes zoonotiques, émergents ou non (ils sont porteurs de 250 agents zoonotiques au total dont 50 agents pathogènes émergents), il ressort toutefois que plus de $40 \%$ des agents infectieux impliquant au moins trois hôtes non humains sont émergents ou réémergents. Cette dernière observation suggère que la capacité d'émergence d'un agent pathogène croit de façon proportionnelle avec le nombre d'hôtes potentiels de cet agent (Woolhouse et Gowtage-Sequeria 2005).

Tableau 2. Les conditions favorisant l'émergence des maladies infectieuses classées par ordre décroissant d'importance (Woolhouse et Sequeria 2005).

\begin{tabular}{|c|c|c|}
\hline \begin{tabular}{|c|} 
Rang \\
d'importance \\
\end{tabular} & Cause favorisante & En relation avec \\
\hline 1 & $\begin{array}{c}\text { Modifications liées à l'agriculture } \\
\text { et à l'exploitation du sol }\end{array}$ & Environnement \\
\hline 2 & $\begin{array}{l}\text { Changements démographiques } \\
\text { et sociologiques }\end{array}$ & Environnement \\
\hline 3 & Dégradation des conditions sanitaires & $\begin{array}{l}\text { Environnement } \\
\text { Hôte } \\
\text { Fnvironnement }\end{array}$ \\
\hline 4 & Les infections liées aux soins médicaux & $\begin{array}{l}\text { Environnement } \\
\text { Hôte } \\
\text { Pathogène }\end{array}$ \\
\hline 5 & $\begin{array}{l}\text { Modifications intrinsèques des agents pathogènes } \\
\text { (acquisition de résistances, virulence accrue ...) }\end{array}$ & Pathogène \\
\hline 6 & $\begin{array}{l}\text { Modifications des habitudes de consommation } \\
\text { (infections hydriques ou alimentaires) }\end{array}$ & Hôte \\
\hline 7 & Les voyages et les échanges commerciaux & Hôte \\
\hline 8 & Les changements climatiques & Environnement \\
\hline
\end{tabular}

tableau 2), tout au moins jusqu'à présent, car il reste très difficile d'établir une relation directe entre le réchauffement général de la planète (lui-même contesté par certains spécialistes, de moins en moins nombreux cependant au fil de l'accumulation des chiffres et des faits (Hansen et al 2006)) et l'émergence de maladies infectieuses nouvelles.

En hiérarchisant ces conditions, il est possible de montrer que les conditions liées à l'environnement sont prépondérantes $(57 \%)$, loin devant les conditions d'émergence liées à l'hôte (27\%) ou à l'agent pathogène lui-même (16\%, figure 1) ; ce schéma montre clairement le caractère non inéluctable de l'apparition de maladies nouvelles liées notamment aux changements génétiques des agents infectieux et pointe plus clairement le rôle important des activités humaines dans 1'apparition de maladies nouvelles.

\section{1 / Les émergences liées à l'en- vironnement}

Nous venons de voir que les émergences liées aux changements environnementaux sont les plus fréquentes ; pour illustrer le rôle des activités humaines sur l'incidence des maladies infectieuses, une récente étude a montré une augmentation significative de l'incidence des maladies diarrhéiques (en particulier celles dues à Escherichia coli, aux rotavirus et à Giardia qui ne sont certes pas des pathogènes émergents au sens strict du terme mais dont l'incidence a augmenté) à la suite de l'ouverture d'une nouvelle route au milieu de la forêt équatoriale en Equateur, Amérique du Sud (Eisenberg et al 2006). Cette observation rejoint d'autres études liées à des constructions de barrages ou d'oléoducs (voir ainsi le site de l'OMS à ce propos sur des études d'impact sur la santé ou «heath impact assessment» : www.who.int/hia/examples/en) ou d'autres observations montrant que la modification d'un écosystème (une route nouvelle par exemple) peut générer des contacts entre l'Homme et la faune sauvage, à l'origine d'émergences liées à des franchissements de barrières d'espèces. C'est le cas des virus de l'immunodéficience acquise (VIH 1 et 2) dans le passé (Heeney et al 2006) ou du simian foamy virus, un autre rétrovirus retrouvé récemment chez des chasseurs de singes en Afrique de l'Ouest (Foley et al 2005, Wolfe et al 2004, 2005). 
Figure 1. Poids relatif (en pourcentage) des trois déterminants de l'émergence des maladies infectieuses*.
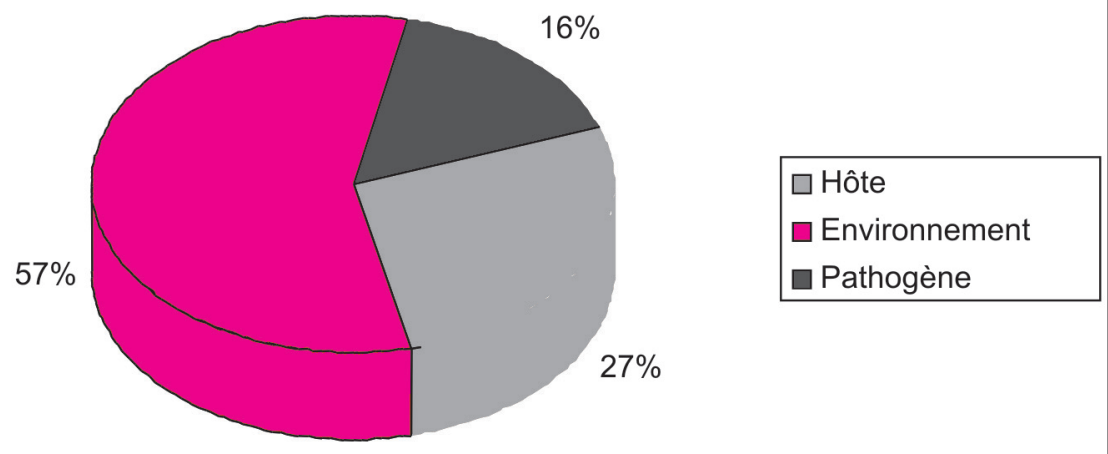

* Les pourcentages ont été calculés en appliquant pour chaque condition favorisante du tableau 1 un barème de points décroissant selon l'ordre d'importance (ainsi la condition 1 liée à l'environnement a un poids de 8 points et ainsi de suite, puis 7 pour la condition 2, etc.)

Il est important aussi de rappeler que les guerres ou guérillas restent un facteur très favorisant de l'émergence ou plus fréquemment encore de la réémergence des maladies infectieuses, par l'écroulement des conditions d'hygiène minimales et la disparition des services de santé ou de contrôle des aliments.

Si les changements liés aux conditions climatiques ne figurent pas parmi les causes premières de l'émergence ou de la réémergence, les nombreux experts dépêchés pour se pencher sur ce sujet s'accordent pour dire que (i) les changements climatiques vont accroître les maladies prévalentes déjà connues telles que les maladies cardiovasculaires, respiratoires ou liées à la malnutrition (Patz et al 2005) et (ii) le risque d'émergence de nouvelles maladies infectieuses ira croissant dans les années à venir si la tendance climatique continue (Albina et al 2005). De nombreuses évaluations des risques ont ainsi été réalisées, soulignant le besoin de réactualiser périodiquement ces données en tenant compte de l'acquisition des nouvelles observations sur les évolutions des climats, du développement des connaissances sur les interactions entre le changement climatique, la biologie des vecteurs (dont les arthropodes) et les maladies animales transmissibles, et de leur confrontation avec les évolutions d'incidence constatées sur le terrain (Gauchard 2005).

\subsection{Les émergences liées à l'agent pathogène}

L'ampleur d'une épidémie ou d'une épizootie liée à un agent pathogène émergent est fonction du nombre initial de cas $\left(\mathrm{I}_{0}\right)$ et du coefficient $R_{0}$ (= «basic reproduction number $»)$, qui va dépendre de la capacité de l'agent pathogène à se transmettre de personne à personne dans le cas d'un agent infectieux humain, et/ou de la dynamique de transmission via un vecteur (à l'exemple des arboviroses). $R_{0}$ mesure le nombre moyen d'infections secondaires générées par un individu infecté au sein d'une population d'individus sensibles (Antia et al 2003). Ainsi un foyer de rage humaine restera limité (lié à un facteur $R_{0}$ du virus de la rage proche de 0 ) alors qu'une épidémie de grippe liée à un passage réussi chez l'Homme d'un virus d'Influenza Aviaire (IA) hautement pathogène (passage d'un $R_{0}$ inférieur à 1 à un $R_{0}$ élevé et supérieur à 1) peut devenir dramatique, au point de parler dans ces circonstances de pandémie à l'image de l'épisode de grippe espagnole en 1918 ; dans ce cas, il a été démontré que le virus d'IA avait subi des modifications génétiques susceptibles d'expliquer sa persistance et son extrême virulence pour 1'Homme (Taubenberger et Morens 2006).

Les émergences liées à des modifications de l'agent pathogène sont donc liées à des modifications du génome de l'agent infectieux :

- par acquisition de gènes de virulence ou de résistance aux antibiotiques à l'exemple de l'augmentation du pouvoir toxinogène de souches de Clostridium difficile devenu émergent dans certains pays (Warny et al 2005) ;

- par une perte de gène(s) d'avirulence (bien décrit dans le monde des nématodes parasites des végétaux et pour certaines bactérioses) ;

- ou par réarrangement de gènes à l'origine de «superbugs» comme le virus de la grippe espagnole de 1918 (Tumpey et al 2005).

\section{3 / Les émergences liées à l'hôte}

Nous avons vu que la multiplicité des hôtes pour les agents pathogènes accroît la probabilité d'émergence et la facilité à franchir la barrière d'espèce notamment chez l'Homme.

Les conditions liées à l'hôte favorisant l'émergence englobent aussi (i) les changements d'habitudes alimentaires et la recherche d'aliments exotiques, avec les risques d'importation d'agents infectieux inconnus jusqu'alors dans le pays importateur et (ii) le développement des voyages à titre touristique (écotourisme en particulier) ou professionnel en relation avec la mondialisation. Ces déplacements peuvent mettre en contact des individus «naïfs» avec des agents pathogènes locaux ou entraîner l'introduction de vecteurs dans le pays de retour, à l'origine, par exemple de foyers de paludisme autour des aéroports dans des pays indemnes.

Les progrès de la médecine ont généré a contrario des «populations à risque accru» tels que les hémophiles ou les transplantés, rendant ces individus plus sensibles aux agents infectieux y compris émergents.

Enfin certains individus dits «super excréteurs» («superspreaders») semblent jouer un rôle épidémiologique important dans la propagation d'une épidémie ou d'une épizootie : cela conduit à considérer les variations individuelles afin de bien comprendre et/ou prédire le déroulement d'une épidémie, comme cela a pu être démontré lors de l'épidémie liée au virus émergent du SRAS en Chine (Lloyd-Smith et al 2005, Shen et al 2004). 


\section{4 / Deux exemples récents de maladies infectieuses émergentes : le SRAS et la bluetongue}

\section{1 / Le SRAS}

Le Syndrome Respiratoire Aigu Sévère ou SRAS (ou SARS en anglais pour «severe acute respiratory syndrome») représente la première importante épidémie du $21^{\text {ème }}$ siècle dont l'origine était inconnue et, à ce titre, peut être considérée comme l'archétype de la maladie infectieuse émergente. Les premiers signes de l'épidémie sont apparus en novembre 2002 dans la province du Guangdong en Chine du Sud, et ont été portés à la connaissance du public en février 2003. L'épidémie a été sous contrôle en juillet 2003, après avoir touché 33 pays sur les cinq continents, causant plus de 700 morts parmi les quelques 8000 individus infectés par ce nouvel agent pathogène. Quatre cas isolés et peu sévères ont été repérés fin 2003 et début 2004. La maladie était essentiellement focalisée sur le tractus respiratoire avec un syndrome évoquant une forte grippe ; la mortalité a surtout été importante chez les personnes âgées. Il semble que la dissémination mondiale de la maladie ait été due à un seul cas passé par un hôtel international de Hong-Kong.

Malgré quelques retards et quelques fausses pistes, la mobilisation de la communauté scientifique a été rapide et exemplaire puisque l'agent pathogène, un nouveau virus de la famille des Coronaviridae appelé «SARS coronavirus» (SARS-CoV), a été identifié quelques mois après le début de l'épidémie. L'origine de ce coronavirus, différent des coronavirus connus jusqu'à présent chez l'homme, restait en revanche inconnue et des enquêtes épidémiologiques ont alors été mises en place pour déterminer son origine. Très rapidement, ces enquêtes sérologiques ont mis en avant l'importance des marchés d'animaux vivants destinés à la consommation humaine. En particulier, des anticorps anti-SARS-CoV ont été détectés régulièrement chez la civette («masked palm civet»; Paguma larvata), de façon plus anecdotique chez d'autres animaux comme le chien viverrin (Nyctereutes procyonoides) ou le blaireau-furet (Melogale moschata), et chez des personnes fréquentant ces marchés. L'attention des chercheurs s'est focalisée sur la civette puisqu'un virus proche du SARS-CoV a été identifié, suggérant que l'Homme se soit contaminé au contact de la civette porteuse de ce coronavirus, capable ensuite de se transmettre de personne à personne. Cette civette est un mammifère sauvage arboricole, carnivore de la famille des viverridés mais au régime très frugivore. Cependant, toutes les pièces du puzzle n'étaient pas en place pour conclure que la civette était le réservoir naturel du virus : il manquait en particulier une véritable dissémination du virus au sein des populations de civettes. La mise en évidence de coronavirus proches mais non identiques chez des chauves-souris tend désormais à suggérer que le réservoir naturel du virus soit plus vraisemblablement des chauves-souris appartenant notamment aux genres Rhinolophus et Miniopterus (Li et al 2005), mais le processus de transmission vers l'homme reste à éclaircir. Les investigations chez les populations de chauves-souris ont montré que $84 \%$ de ces animaux étaient porteurs d'anticorps antiSARSlike-CoV, avec une distribution géographique très large des chauvessouris positives. Cela reste bien sûr une hypothèse à l'heure présente, mais elle se renforce devant l'absence d'autres réservoirs identifiés. Si cette hypothèse se confirmait, elle illustrerait parfaitement les conditions de l'émergence d'un nouvel agent pathogène en faisant apparaître des modifications en quatre étapes, surtout liées à l'agent pathogène (capacité de franchissement de la barrière d'espèce), mais aussi à des modifications liées à l'environnement comme l'intensification de la production de la civette (fermes de civettes) pour la consommation humaine :

1) Contact entre espèces différentes (en l'occurrence entre chauves-souris et civettes) ;

2) Transmission inter-espèces (1 ${ }^{\mathrm{er}}$ franchissement de la barrière d'espèce) ;

3) Persistance chez l'hôte intermédiaire infecté et adaptation du virus à cette espèce ;

4) Transmission à l'Homme (2ème franchissement de la barrière d'espèce), via les contacts lors de la manipulation des animaux et de la viande, d'un virus dont les caractéristiques de départ ont varié et désormais capable de persister chez l'Homme conduisant à l'épidémie de SRAS (Wang et al 2006).
Cette histoire naturelle du SRAS montre que les chauves-souris représentent une source importante de maladies infectieuses, puisqu'à côté du SARSlike-Cov les chauve-souris peuvent héberger d'autres virus comme les henipavirus, le virus Menangle, le paramyxovirus Tioman ou des lyssavirus ${ }^{1}$ (Picard-Meyer et al 2006).

\section{2 / La fièvre catarrhale ovine}

La bluetongue était une maladie exotique pour l'Europe jusqu'en 2000, date à laquelle cette maladie animale a émergé dans les pays européens du pourtour méditerranéen, et en Corse en particulier pour la France (Zientara et al 2002). La bluetongue, qui sévissait un peu partout dans le Monde et notamment dans les pays africains du Maghreb, est due à un orbivirus proche du virus de la peste équine et est transmise par la piqûre d'un moucheron du genre Culicoides ; la maladie affecte les ruminants, tout particulièrement les ovins et dans une moindre mesure les bovins, et n'est pas transmissible à l'Homme. Le virus de la bluetongue (le BTV) compte 24 sérotypes et l'Europe a connu des épizooties dues aux sérotypes 1, 2, 4, 9 et 16. En Corse, seuls les sérotypes 2,4 et 16 ont été mis en évidence. Les conséquences de la maladie, une fois l'épisode aigu passé (avec une morbidité importante et une mortalité relativement faible) concernent surtout les restrictions de circulation des animaux afin d'éviter la propagation de la maladie (MacLachlan et Osburn 2006), en application des recommandations faites par l'Organisation Mondiale de la Santé Animale (ex Office International des Epizooties, OIE) qui a classé la bluetongue parmi les maladies animales prioritaires au même titre que la fièvre aphteuse ou l'influenza aviaire.

La brutale apparition de la bluetongue dans les pays du Bénélux, en Allemagne puis en France durant l'été 2006 est l'illustration nette de la capacité des agents infectieux à apparaître là où $a$ priori ils n'étaient pas attendus. En effet, les experts prévoyaient plutôt une émergence de la bluetongue dans le sud de la métropole, puisque l'Italie et l'Espagne déclaraient régulièrement de nouveaux cas. Le sérotype en cause dans l'épisode toujours en cours du Bénélux est le sérotype 8 inconnu jusqu'à présent y compris dans les épisodes autour du bassin méditerranéen (Enserink 2006, Toussaint et al 2006).

1 http://www2a.cdc.gov/podcasts/media/pdf/EIDtranscript11-8-06 Bats SARS.pdf 
Ces épisodes illustrent parfaitement la notion d'émergence pour un agent infectieux connu, à la différence du SARS-CoV, avec une apparition dans des régions indemnes. Les causes de ces émergences dans les pays méditerranéens et en Europe du Nord sont inconnues et seules des hypothèses sont mises en avant pour expliquer ce saut du BTV :

- transport illicite ou non d'animaux infectés mais non décelés dans les régions indemnes?

- transfert de moucherons infectés via des supports inertes comme des fleurs, des légumes, etc. ?

- modification de la répartition du vecteur (notamment concernant celle des Culicoïdes vecteurs du virus de la bluetonque dans le bassin méditerranéen) à rapprocher d'un effet du réchauffement climatique ?

- ou combinaison de différents facteurs?

\section{5 / Les maladies infectieuses émergentes : se préparer pour le futur}

Il est évident qu'il sera difficile voire impossible de prévenir les émergences qui, par définition, sont imprévisibles ou faiblement prévisibles. En revanche, il est indispensable de se préparer à mieux identifier les possibles émergences ou réémergences afin d'en réduire considérablement les conséquences sur la santé humaine, la santé et le bien-être de l'animal, sur l'économie, etc. Dans ce cadre, les autorités publiques et sanitaires nationales et européennes ont d'ores et déjà réagi en réformant le système de veille sanitaire avec la création, entre autres, de l'InVS (Institut de Veille Sanitaire) et de l'Agence Française de Sécurité Sanitaire des Aliments (AFSSA) en France, et de l'EFSA (European Food Safety Authority) et de l'ECDC (European Center for Disease Prevention and Control) en Europe. Ces différentes structures sont destinées à mieux évaluer les risques (avec en particulier la séparation de la gestion de ces mêmes risques) afin de fournir aux décideurs des arguments scientifiquement validés pour mettre en place telle ou telle mesure de police sanitaire ou prophylactique.

En France, le récent épisode du chikungunya dans l'île de la Réunion (Josseran et al 2006) a aussi conduit les autorités publiques à se doter d'une cellule de recherche et d'appui scientifique sur les maladies infectieuses émergentes (CP-MIE), placée sous l'autorité du Ministère de la Recherche en relation avec les autres ministères concernés (Santé, Agriculture...). Cette cellule permanente doit agir selon trois niveaux d'intervention :

- accompagner l'urgence notamment sur les crises actuelles dues au virus du chikungunya ou de l'influenza aviaire ;

- amplifier l'effort de recherche sur le moyen et long terme (recherche fondamentale et finalisée) ;

- anticiper les crises futures par une meilleure coordination permanente.

Cette cellule a ainsi déjà (i) défini plusieurs axes stratégiques de recherches à développer ou renforcer en mettant en réseau les différents acteurs français de la recherche dans les domaines concernés (tableau 3 ), (ii) contribué à renforcer l'effort de financement de la recherche sur la problématique «maladies émergentes» et (iii) créé un contexte pour développer les échanges et la collaboration au niveau européen et international. Des initiatives similaires se mettent aussi en place dans d'autres pays ou groupes de pays avec l'idée à terme de construire un réseau mondial capable de réagir rapidement à toute émergence ou réémergence sérieuse apparue sur n'importe quel point de la planète (King et al 2006, Kuiken et al 2005).

Tableau 3. Les 7 actions de recherches prioritaires définies par la cellule permanente sur les maladies infectieuses émergentes (CP-MIE).

\begin{tabular}{|c|c|}
\hline Action à mener & $\begin{array}{c}\text { Organisme(s) } \\
\text { coordonateur(s) }\end{array}$ \\
\hline $\begin{array}{c}\text { Création d'un observatoire des données épidémiologiques } \\
\text { (maladies humaines et animales) } \\
\text { Renforcement des moyens de caractérisation } \\
\text { des agents infectieux } \\
\text { Coordination des compétences sur les processus } \\
\text { physiopathologiques } \\
\text { Mise en place de nouvelles stratégies diagnostiques, } \\
\text { thérapeutiques et vaccinales } \\
\text { Surveillance de la dynamique et de l'évolution } \\
\text { des vecteurs } \\
\text { Mise en place de méthodes prophylactiques compatibles } \\
\text { avec l'environnement } \\
\text { Etude du comportement des acteurs } \\
\text { et modalités de gestion des crises }\end{array}$ & $\begin{array}{l}\text { InVS }{ }^{a} \text { et AFSSA } \\
\text { Institut Pasteur } \\
\text { INSERM } \\
\text { CNRS }^{\mathrm{d}} \text { et } \text { CEA }^{\mathrm{e}} \\
\text { IRD }^{f} \text { et } \text { CIRAD }^{\mathrm{g}} \\
\text { INRA }^{\mathrm{h}} \\
\text { CNRS }^{\mathrm{d}}\end{array}$ \\
\hline
\end{tabular}

alnVS : Institut de Veille Sanitaire ; bAFSSA : Agence Française de Sécurité Sanitaire des Aliments ; CINSERM : Institut National de la Santé et de la Recherche Médicale ; dCNRS : Centre National de Recherche Scientifique ; ${ }^{e} C E A$ : Commissariat à l'Energie Atomique ; fIRD: Institut de Recherche pour le Développement ; gCIRAD : Centre de coopération Internationale en Recherche Agronomique pour le Développement; hINRA : Institut National de la Recherche Agronomique. 
al 2006) ; ainsi 13 au moins des 39 agents infectieux décrits récemment sont impliqués dans des syndromes chroniques, comme par exemple la découverte récente d'un syndrome pseudo-poliomyélitique dû à l'infection par le virus West Nile (Sejvar et al 2005);

- de mettre en place des systèmes de surveillance et d'alerte des maladies animales émergentes et potentiellement zoonotiques chez (i) les animaux domestiques via le réseau des vétérinaires sanitaires et (ii) dans la faune sauvage, réservoir potentiel de nombreuses maladies infectieuses émergentes (Chomel et al 2007, Polley 2005, Vourc'h et al 2006) ;

\section{- d'encourager :}

- une communication accrue entre le monde médical et le monde vétérinaire, - et la multidisciplinarité.
Bien sûr, ce combat contre les maladies infectieuses émergentes passe aussi et surtout par une meilleure coordination et coopération entre pays (et notamment avec les pays du Sud) afin d'accéder à la notion de «global network» sous l'égide des organisations internationales comme l'OIE, la FAO (Food and Agricultural Organization) et l'OMS (Organisation mondiale de la Santé) (Domenech et al 2006, Heeney 2006).

\section{Références}

Albina E., André-Fontaine G., Armengaud M., Dreyfuss G., Dufour B., Duvallet G., Moutou F., Rodhain F., Zientara S., 2005. Rapport sur l'évaluation du risque d'apparition et de développement des maladies animales compte tenu d'un éventuel réchauffement climatique, AFSSA (Ed), Maisons-Alfort, France, 1-78.

Antia R., Regoes R.R., Koella J.C., Bergstrom C.T., 2003. The role of evolution in the emergence of infectious diseases. Nature, 426, 658-661.

Blaga R., Durand B., Antoniu S., Gherman C., Cozma V., Boireau P., 2007. Dramatically increase of human trichinellosis incidence in Romania over the last 25 years: impact of political changes or regional food habits? Am. J. Trop. Med. Hyg., 76 (5), 983-986.

Chomel B.B., Belotto A., Meslin F.X., 2007. Wildlife, exotic pets, and emerging zoonoses. Emerg. Infect. Dis., 13, 6-11.

Domenech J., Lubroth J., Eddi C., Martin V., Roger F., 2006. Regional and international approaches on prevention and control of animal transboundary and emerging diseases. Ann. N.Y. Acad. Sci., 1081, 90-107.

Eisenberg J.N., Cevallos W., Ponce K., Levy K., Bates S.J., Scott J.C., Hubbard A., Vieira N., Endara P., Espinel M., Trueba G., Riley L.W., Trostle J., 2006. Environmental change and infectious disease: How new roads affect the transmission of diarrheal pathogens in rural Ecuador. Proc. Natl. Acad. Sci., 103, 19460-19465.

Enserink M., 2006. Emerging infectious diseases. During a hot summer, bluetongue virus invades northern Europe. Science, 313, 12181219.

Foley J.A., DeFries R., Asner G.P., Barford C., Bonan G., Carpenter S.R., Chapin F.S., Coe M.T., Daily G.C., Gibbs H.K., Helkowski J.H., Holloway T., Howard E.A., Kucharik C.J., Monfreda C., Patz J.A., Prentice I.C., Ramankutty N., Snyder P.K., 2005. Global consequences of land use. Science, 309, 570-574.

Gauchard F., 2005. Evaluation du risque d'apparition et de développement de maladies animales compte tenu d'un éventuel réchauffement climatique. Bull. Epidémiol., 19, 1-3.

Gould E.A., Higgs S., Buckley A., Gritsun T.S., 2006. Potential arbovirus emergence and implications for the United Kingdom. Emerg. Infect. Dis., 12, 549-555.

Hansen J., Sato M., Ruedy R., Lo K., Lea D.W., Medina-Elizade M., 2006. Global temperature change. Proc. Natl. Acad. Sci., 103, 14288-14293.
Heeney J.L., 2006. Zoonotic viral diseases and the frontier of early diagnosis, control and prevention. J. Int. Med., 260, 399-408.

Heeney J.L., Dalgleish A.G., Weiss R.A., 2006. Origins of HIV and the evolution of resistance to AIDS. Science, 313, 462-466.

Josseran L., Paquet C., Zehgnoun A., Caillere N. Le Tertre A., Solet J.L., Ledrans M., 2006. Chikungunya disease outbreak, Reunion Island. Emerg. Infect. Dis., 12, 1994-1995.

King D.A., Peckham C., Waage J.K., Brownlie J., Woolhouse M.E., 2006. Epidemiology, infectious diseases: preparing for the future. Science, 313, 1392-1393.

Kuiken T., Leighton F.A., Fouchier R.A., LeDuc J.W., Peiris J.S., Schudel A., Stohr K., Osterhaus A.D., 2005. Public health. Pathogen surveillance in animals. Science, 309, 1680-1681.

Li W., Shi Z., Yu M., Ren W., Smith C., Epstein J.H., Wang H., Crameri G., Hu Z., Zhang H., Zhang J., McEachern J., Field H., Daszak P., Eaton B.T., Zhang S., Wang L.F., 2005. Bats are natural reservoirs of SARS-like coronaviruses. Science, 310, 676-679.

Lloyd-Smith J.O., Schreiber S.J., Kopp P.E., Getz W.M., 2005. Superspreading and the effect of individual variation on disease emergence. Nature, 438, 355-359.

MacLachlan N.J., Osburn B.I., 2006. Impact of bluetongue virus infection on the international movement and trade of ruminants. J. Am. Vet. Med. Assoc., 228, 1346-1349.

O'Connor S.M., Taylor C.E., Hughes J.M., 2006. Emerging infectious determinants of chronic diseases. Emerg. Infect. Dis., 12, 10511057.

Patz J.A., Campbell-Lendrum D., Holloway T., Foley J.A., 2005. Impact of regional climate change on human health. Nature, 438, 310-317.

Picard-Meyer E., Barrat J., Tissot E., Verdot A., Patron C., Barrat M.J., Cliquet F., 2006. Bat rabies surveillance in France, from 1989 through May 2005. Dev. Biol., 125, 283-288.

Polley L., 2005. Navigating parasite webs and parasite flow: emerging and reemerging parasitic zoonoses of wildlife origin. Int. J. Parasitol., 35 1279-1294.

Sejvar J.J., Bode A.V., Marfin A.A., Campbell G.L., Ewing D., Mazowiecki M., Pavot P.V., Schmitt J., Pape J., Biggerstaff B.J., Petersen L.R., 2005. West Nile virus-associated flaccid paralysis. Emerg. Infect. Dis., 11, 1021-1027.

Shen Z., Ning F., Zhou W., He X., Lin C., Chin D.P., Zhu Z., Schuchat A., 2004.
Superspreading SARS events, Beijing, 2003. Emerg. Infect. Dis., 10, 256-260.

Taubenberger J.K., Morens D.M., 2006. 1918 Influenza: the mother of all pandemics. Emerg. Infect. Dis., 12, 15-22.

Toma B., Thiry E., 2003. Qu'est ce qu'une maladie émergente ? Epidémiol. Santé Anim., 44, 1-11.

Toussaint J.F., Vandenbussche F., Mast J., De Meester L., Goris N., Van Dessel W., Vanopdenbosche E., Kerkhofs P., De Clercq K., Zientara S., Sailleau C., Czaplicki G., Depoorter G., Dochy J.M., 2006. Bluetongue in Northern Europe. Vet. Rec., 159, 327.

Tumpey T.M., Basler C.F., Aguilar P.V., Zeng H., Solorzano A., Swayne D.E., Cox N.J., Katz J.M., Taubenberger J.K., Palese P., Garcia-Sastre A., 2005. Characterization of the reconstructed 1918 Spanish influenza pandemic virus. Science, 310, 77-80.

Vourc'h G., Bridges V.E., Gibbens J., De Groot B.D., McIntyre L., Poland R., Barnouin J., 2006. Detecting emerging diseases in farm animals through clinical observations. Emerg. Infect. Dis., 12, 204-210.

Wang L.F., Shi Z., Zhang S., Field H., Daszak P., Eaton B.T., 2006. Review of bats and SARS Emerg. Infect. Dis., 12, 1834-1840.

Warny M., Pepin J., Fang A., Killgore G., Thompson A., Brazier J., Frost E., McDonald L.C., 2005. Toxin production by an emerging strain of Clostridium difficile associated with outbreaks of severe disease in North America and Europe. Lancet, 366, 1079-1084.

Wolfe N.D., Daszak P., Kilpatrick A.M., Burke D.S., 2005. Bushmeat hunting, deforestation, and prediction of zoonoses emergence. Emerg. Infect. Dis., 11, 1822-1827.

Wolfe N.D., Switzer W.M., Carr J.K., Bhullar V.B., Shanmugam V., Tamoufe U., Prosser A.T. Torimiro J.N., Wright A., Mpoudi-Ngole E., McCutchan F.E., Birx D.L., Folks T.M., Burke D.S., Heneine W., 2004. Naturally acquired simian retrovirus infections in central African hunters. Lancet, 363, 932-937.

Woolhouse M.E., Gowtage-Sequeria S., 2005. Host range and emerging and reemerging pathogens. Emerg. Infect. Dis., 11, 1842-1847.

Zientara S., Sailleau C., Dauphin G., Roquier C., Remond E.M., Lebreton F., Hammoumi S. Dubois E., Agier C., Merle G., Breard E., 2002. Identification of bluetongue virus serotype 2 (Corsican strain) by reverse-transcriptase PCR reaction analysis of segment 2 of the genome. Vet. Rec., 150, 598-601. 


\title{
Résumé
}

Les maladies infectieuses émergentes, qu'elles soient animales, humaines ou zoonotiques, sont devenues une composante essentielle dans le paysage sanitaire mondial. Qu'elles soient inconnues jusqu'alors pour les vraies émergences ou simplement qu'elles réapparaissent après plusieurs années voire des décennies de silence dans le cas des réémergences, les maladies infectieuses émergentes ou réémergentes sont caractérisées par une augmentation brutale de leur incidence et par une incertitude sur l'ampleur du phénomène. Dans cette revue, les conditions de l'émergence sont présentées et hiérarchisées en fonction des connaissances actuelles, selon que ces conditions soient liées à l'environnement, à l'hôte (ou les hôtes) ou à l'agent infectieux lui-même. Deux exemples sont présentés pour illustrer le propos : d'une part une maladie humaine nouvelle, le SRAS avec un ou des réservoirs animaux et une maladie strictement animale, la fièvre catarrhale ovine ou bluetongue apparue récemment en Europe. Face à ces émergences ou réémergences, les différents acteurs, chercheurs et autorités sanitaires, doivent s'organiser y compris au niveau mondial afin de prévenir ou au mieux de bien se préparer à ces émergences de maladies infectieuses.

\begin{abstract}
Emergence of animal and human infectious diseases

Emerging infectious diseases are becoming more and more important throughout the world. Emerging or reemerging infectious diseases are characterized by a high and rapid increase of their incidence and by the uncertainty about their exact impact on humans or on animals or on both in the case of zoonoses. In this paper, the conditions or «drivers» of emergence and reemergence are presented and classified according to three parameters: the environment, host(s) and pathogen. Two examples of emerging diseases are presented: the first one concerns SARS in humans with the identification of potential animal reservoirs and the second one concerns the bluetongue virus, which has emerged recently in ruminants in Europe. The early identification, control and prevention of emerging and reemerging infectious diseases lies not only with clinicians and public health experts, but importantly with veterinarians, animal scientists and wildlife ecologists; surveillance of perturbations in ecosystems and surveillance of the animal and human health continuum must be done at the local, national and international levels to ensure that we are well prepared for future emerging threats.
\end{abstract}

PÉPIN M., BOIREAU P., BOUÉ F., CASTRIC J., CLIQUET F., DOUZAL Y., JESTIN A., MOUTOU F., ZIENTARA S., 2007. Emergence des maladies infectieuses animales et humaines. INRA Prod. Anim., 20, 199-206. 
